\title{
A distributed architecture to support infomobility services
}

\author{
Claudia Canali, Riccardo Lancellotti \\ Department of Information Engineering \\ University of Modena and Reggio Emilia \\ \{canali.claudia, lancellotti.riccardo\}@unimore.it
}

\begin{abstract}
The growing popularity of mobile and location aware devices allows the deployment of infomobility systems that provide access to information and services for the support of user mobility. Current systems for infomobility services assume that most information is already available on the mobile device and the device connectivity is used for receiving critical messages from a central server. However, we argue that the next generation of infomobility services will be characterized by collaboration and interaction among the users, provided through realtime bidirectional communication between the client devices and the infomobility system.

In this paper we propose an innovative architecture to support next generation infomobility services providing interaction and collaboration among the mobile users that can travel by several different transportation means, ranging from cars to trains to foot. We discuss the design issues of the architecture, with particular emphasis on scalability, availability and user data privacy, which are critical in a collaborative infomobility scenario.
\end{abstract}

\section{Introduction}

The increasing popularity of mobile systems with network capabilities and the advent of affordable GPSequipped devices bring new interesting opportunities for the deployment of infomobility services. Infomobility provides mobile users with location-aware information and services that aim to help the users throughout their trip. The possible services are extremely heterogeneous and range from the simple notification of points of interest (POIs), such as museums or restaurants on the user's route, to real-time alert of critical events, such as car accidents or train delays, which can represent a problem for the mobile user that is traveling by different transportation means (e.g., car, train, bus, foot).
However, even the most modern navigation systems do not fully exploit the potential of infomobility. In particular, the opportunities introduced by network connections are in most cases neglected and the navigation system relies on a priori downloaded data on the devices.

One of the major issues of navigation systems based on a priori data upload is the inability to provide always up-to-date maps that take into account changing route conditions due to issues such as traffic jams, road construction or transportation delays [6].

For example, navigation maps cannot be continuously updated because this could require a significant amount of information processing and tranfer resulting in high costs for the customers. Moreover, next generation infomobility services will exploit some form of collaboration between the users and the system itself, by allowing users to exchange geo-referenced notifications. This collaboration allows the deployment of innovative services: for example, to inform the user about occurring events (e.g., transportation delays or car accidents) or to exchange in real-time POIs among the users. All these services cannot be provided through previously downloaded maps, but require a bidirectional, real-time information exchange between the user and the infomobility architecture.

The main contribution of this paper is the proposal of a novel architecture for infomobility that supports bidirectional, real-time information exchange to allow highly interactive and collaborative services, such as geo-referenced bulletin boards. Indeed, the users are able to communicate critical information or POIs to the system as they travel; the system will elaborate the received information and continuously inform the users of changing conditions. Furthermore, the proposed architecture allows the users to store on their device only the information required for a given trip, without the need to download or update large map sets. The proposed infomobility scenario with highly interactive mobile users and collaborative services introduces issues that are not common in today's infomobility. The pro- 
posed architecture guarantees scalability and availability to support the continuous information exchange between the users and the system. Moreover, the management of sensitive information, such as the user position and identity, introduces privacy issues that must be addressed by the infomobility system. These are the key motivations for our proposal of a new architectural solution for infomobility services. To the best of our knowledge this is the first paper addressing the issues of designing large scale distributed infrastructures for supporting advanced infomobility services.

The remainder of this paper is organized as follows. Section 2 discusses the related work and outlines the innovative characteristics of our proposal. Section 3 describes the typical services provided by infomobility systems and the resulting issues to be addressed. Section 4 provides an overview of the proposed architecture, while Section 5 describes in deeper details the architecture elements. Finally, Section 6 concludes the paper with some final remarks.

\section{Related work}

Even if pervasive computing represents a very popular topic, the proposal of advanced applications and architectures oriented to infomobility support has been only partially explored by the research community $[13$, $15,5]$. The study proposed in [13] focuses on the interaction between mobile users and infomobility services, proposing XUL-based interfaces that have some similarities with the Web-based approach proposed in this paper. However, the study does not address the architectural issues of scalability, availability and privacy in the deployment of infomobility services. Moraitis et al. [15] propose a middleware for infomobility support based on mobile agents. Architectures based on mobile agents have been proposed in other studies on pervasive computing, however, our proposal follows a different approach based on Web services. In [5] Cole et al. present a middleware that provides mobile users with exhaustive location-aware information through a dynamic binding of different services. However, this proposal does not allow the users to actively interact with the system to communicate real-time feedback necessary to handle on-the-fly events, such as changing road or transportation conditions.

Furthermore, all the above-mentioned studies do not consider the privacy issues involved in accessing location-based services. Indeed, in any infomobility context critical issues arise about how positioning information is stored, managed and released to possibly untrusted services, as pointed out in [3], where the authors formally define the problem of sensitive user information management in location-based services and propose algorithms to prevent user identity disclosure. On the other hand, our proposal preserves the privacy of sensitive data, such as user position and identity.

More interest towards infomobility has been demonstrated by the industry. For example, Google Earth [8] and Google Maps [9] provide an intuitive Web-based interface to access geographic information. However, such systems do not provide support for infomobility services where dynamic geo-referenced information can be communicated to users (e.g., dynamic notification of traffic jams). Even more important, such systems do not allow users to directly interact with the system by notifying real-time events. A similar lack of interactivity is typical of other Web interfaces to GIS, such as Web portals powered by Navteq technology (e.g., map-o-rama Web site [12]), as well as of the most advanced products for navigation, such as the Tom-Tom system [16]. Another limitation of these systems is that they provide route plans only for users moving by car or foot, while our architecture for infomobility services considers the possibility for the users to travel by different transportation means, including trains, buses or subways.

An additional research topic related to our proposal is the field of vehicular networks. Most vehicular networks are specialized ad-hoc networks that allow vehicles to share information while moving. An interesting study [14] proposes the use of vehicular networks to improve driving safety and to provide navigation services to the car passengers. However, the vehicular networks are most suitable in high-density vehicular traffic scenarios such as highways, while our proposal aims to provide a more general approach to infomobility where services can be also exploited in the case of low traffic routes.

\section{Infomobility services and issues}

Infomobility services are extremely heterogeneous. We describe some innovative services to introduce the issues that should be addressed by infomobility support architectures.

Infomobility services are usually related to the management of geo-referenced data. Providing maps for a given location or computing a route between two points (by foot, car or public transportation) are typical examples of infomobility services. Infomobility services often provide additional useful information, such as maps layers containing POIs. However, all these POIs are time-independent, which means that there is no need to update these information often. As a consequence, POIs sharing among the users is usually carried out in forums or newsgroups, where users can download 
the desired maps and upload them on their GPS navigator.

A critical feature of advanced infomobility services is the management of time-dependent geographic information, such as traffic or weather conditions. Even if some systems provide a notification service for traffic jams and car accidents, this notification does not involve any form of interaction of the user towards the system. However, we argue that infomobility will evolve towards sophisticated services where a bidirectional interaction occurs between the clients and the system. This evolution is similar to what occurred to the Web, that evolved from a simple information repository to a system providing services that enable user collaboration. An infomobility architecture allowing bidirectional information exchange is the basis for always upto-date navigation systems that can handle on-the-fly events, such as changing road conditions, thanks to accurate and real-time information. Furthermore, the architecture can be exploited for other value-added applications, such as real-time POIs sharing among the users. Finally, the analysis of the user feedback, including position and (possibly) speed, allows an early detection of traffic jams, queues or public transportation delays.

From an architectural point of view, the support for the above-mentioned services introduces multiple issues to address.

First, it is necessary to provide adequate performance and scalability in a scenario where the user request load is significantly higher with respect to traditional infomobility services due to users interaction and collaboration.

Second, the architecture must guarantee a high level of availability. Indeed, the user relies on the infrastructure to download real-time information updates. As a consequence, a low availability of the architecture has a direct impact on the availability of the offered service, with potential problems for the users.

Third, the presence of personalized services where the user can communicate geo-referenced data introduces the issues related to the privacy of the user. User position and identity are sensitive data that should not be disclosed even in the case of a security problem in the system, such as an attack.

We anticipate that the above-mentioned issues result in a trade-off between distribution for scalability and availability and centralization for privacy reasons. The architecture described throughout the paper aims to address the trade-off through a hybrid approach as discussed in Section 4.

\section{Architecture overview}

The distributed architecture proposed in this paper is based on a two-level organization of nodes, as shown in Figure 1. The first level is composed by locally distributed nodes (cluster) and represents the so-called central system, shown on the right side of Figure 1.

The central system is a key component of the infrastructure that hosts a complex geographical information system (GIS) and additional support databases (e.g., buses/trains schedules and status). Information in the additional support databases can be fed directly by the provider of the infomobility services, or can be retrieved by accessing external data sources (e.g., public transportation company DB). The proposed architecture is flexible enough to act both as a stand-alone supplier of infomobility services and as a broker for heterogeneous information/services supplier. The information managed by the central system is used to compute the best itinerary for each user, given the endpoints of the route and the desired transportation mean.

The second level is based on geographically distributed edge nodes, represented in the left part of Figure 1. Edge nodes are responsible for storing information (maps and other geographical data, such as POIs) related to a specific and limited area, that we will call cell. Furthermore, edge nodes are also responsible for the communications to and from the mobile users (the running man in Figure 1) traveling across the cells.

The services provided by the infomobility system require a significant amount of computational resources. Due to the novelty of infomobility services, there are no universally accepted workload models to be used in the architecture design. However, our experience in distributed systems with high computational requirements suggests that a single node can handle a volume of requests in the order of hundreds of requests per second. The performance of such systems is typically limited by the computational power of the nodes servicing requests and by the throughput (i.e., amount of transactions per seconds) that can be handled by the data storage. In the considered infomobility scenario, the user sends its first requests to the central system and then interacts with the edge nodes of the system at the rate of a request every few seconds. Hence, we can conclude that each node can handle a user population in the order of thousands of users. From these considerations, we can infer that supporting infomobility for a number of citizens in the order of a few million requires an architecture consisting of thousands of nodes. In this scenario, the choice of a two-level architecture is an innovative solution for infomobility that provides significant advantages over completely centralized or completely distributed approaches. 


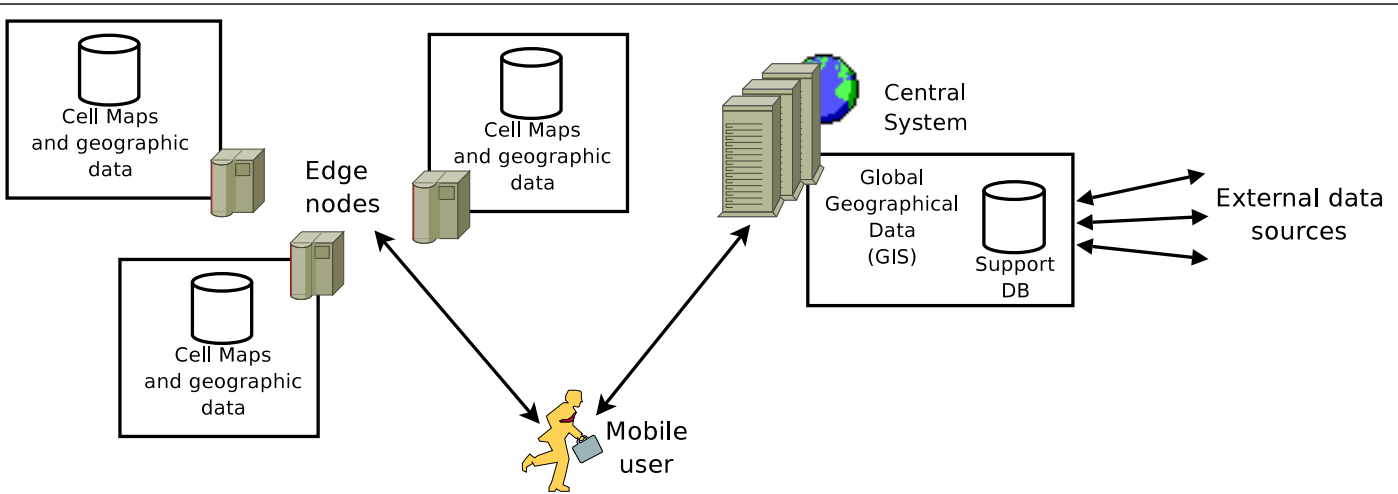

Figure 1. Distributed architecture for Infomobility

A centralized architecture, where only a central cluster of nodes manages every function, is not a suitable solution for the infomobility issues due to inadequate scalability and availability. Indeed, the central system represents a single point of failure that can be taken off-line due to attacks or network failures. Furthermore, even if computational power may be added in a seamless way thanks to clustering techniques, the network connecting the system with the clients may become a bottleneck, thus limiting the scalability of the system. The scalability and reliability limits of centralized architectures are well known in the Web literature [2] and these conclusions are directly applicable to infomobility services. On the other hand, a geographically distributed approach, such as the edge servers used in our architecture, is clearly a better alternative to guarantee scalability and availability. Since each edge node is responsible for a specific cell, the client request traffic for both time-dependent and time-independent information is distributed among the set of edge nodes, thus increasing the system scalability. The highly distributed edge nodes also provide advantages about the system availability. Indeed, a local failure due to power loss, congestion or attack is related to only one or few cells. Simple redundancy techniques (described in deeper detail in Section 5.3) can be used to guarantee system availability even in the case of a failure in a subset of nodes.

On the other hand, a completely distributed architecture introduces significant issues with respect to data consistency and privacy. Preserving data consistency in a scenario characterized by a high number of nodes and highly volatile information is unfeasible [10]. For this reason, it is mandatory to partition information among the nodes to preserve consistency. However, partitioning information hinders the deployment of services that require a global knowledge of the geographical data. The central system supports services such as the computation of routes and the aggregation of ex- ternal data sources (as in the example of public transportation database) that would not be easily provided in the case of a completely distributed architecture.

The completely distributed architecture also introduces issues with respect to data privacy. Recently, the requirements of security and privacy in providing advanced services including personalization and locationawareness features are getting more and more attention $[4,11,3]$. Distributed architectures may be inadequate to guarantee the privacy of sensitive user data, because it is unfeasible to guarantee the same high security standard for every node, especially when the architecture is composed by thousands of nodes. On the other hand, a centralized approach with few controlled locations may provide an higher level of system security through physical access control to the location of the servers and frequent software security checks. In the case of infomobility services, the user physical location is a sensitive information that is exchanged in most interactions. Our approach to guarantee privacy of user location is based on a separation between user identity and location data: the user identity is stored and managed only on the central system, while the only information maintained on the edge nodes is a temporary user access credential that does not allow any direct association with the user identity.

The hybrid approach of the two-level architecture successfully addresses the trade-off between centralization and distribution, because it joins the consistency and security advantages of a centralized architecture for the privacy of sensitive user data with the scalability and availability characteristics of distributed architectures.

\section{Architecture details}

In this section we describe in detail the distributed architecture for infomobility support. To better understand how the architecture may provide infomobility 
services addressing performance, scalability, availability and privacy issues, we first describe the dynamic interaction with the users, then we focus on the main system components.

\subsection{Architecture functioning}

Figure 2 describes how the end user dynamically interacts with the distributed architecture for infomobility. As shown in Figure 2, the user issues a route planning request to the central system (step 1) communicating his current coordinates and final destination. The GIS software on the central system calculates the best route plan for the user based on real-time information updates, that are available thanks to the notifications coming from the edge nodes, as better explained in Section 5.3. The geo-referenced data identifying the route plan are sent back to the user along with additional information (step 2) needed for the following communications between the user and the distributed edge nodes. This information consists of the list of edge nodes responsible for the cells that constitute the selected route (cells $\mathrm{C} 1, \mathrm{C} 2$, and C3 in Figure 2) and the access credentials required to communicate with the edge nodes.

Then, the user starts traveling following the route plan. While crossing a specific cell, the user exchanges information with the corresponding edge node (steps 3, 4 and 5): the edge node informs the user of the possible POIs that are located nearby, while the user periodically communicates his current coordinates. Moreover, the user may notify in an asynchronous way the edge node of particular events he comes across along the route.

The notifications of the traveling users concerning the currently crossed cell can be of two types: information with local relevance, that is information that only concern the covered cell (e.g., new POIs), and critical notifications with global relevance, that may affect the routing planning decisions of the central system (e.g., traffic jams, detours, pile-ups, buses/trains delays). If the received information has a local relevance, the edge node simply stores the data for future notifications to other users. On the other hand, if the information has a global relevance, it has to be communicated to the central system to allow the GIS software to plan routes taking into account the changed road conditions. In this case, the edge node elaborates the information by aggregating it with other notifications, as better described in Section 5.3, and then communicates the information to the central system (step $\mathrm{N})$.

It is worth noting that in the proposed architecture, the communication between the client device and the infrastructure occurs through wireless channels typical of infomobility services, such as GPRS, UMTS or WiMAX connections, to allow the user to access the information from every location along his route. The access to the infomobility services should be provided not only from every location, but also from a large set of client devices. Allowing access from different client platforms increases the user population, thus improving the quality of the offered service especially with respect to the amount of shared information. For this reason we plan to exploit Web services for our architecture. Indeed, Web services are emerging as a standard for interoperability among heterogeneous devices. Furthermore, exploiting popular protocols simplifies the deployment of the infomobility architecture thanks to the availability of reliable and efficient technologies for distributed Web-based systems.

\subsection{The central system}

The central system relies on a GIS software and on additional information database to obtain geo-referenced data that identify a route plan. In the case of a trip by car or by foot the route plan contains a list of the roads composing the path, the distance to cover on each road, directives and coordinates of road connections and junctions, while in the case of a bus trip the plan also contains the information about bus lines, with stops and schedule. This information allows the users to easily follow the suggested itinerary with the help of a simple GPS receiver. It is important to note that our architecture avoids the users to download a priori last-version maps, since the system is able to provide the users with a complete and real-time updated set of information concerning their travel.

The proposed architecture for infomobility support makes use of innovative mechanisms to provide the users with always up-to-date information on the required itinerary. Indeed, the central system relies on real-time updates directly communicated by the user as they travel, thus allowing to consider on-the-fly events like changing road conditions or bus/train delays in the computation of the best route plan.

Another important issue addressed by our architecture for infomobility support is related to preserving privacy in the management of user information. When a user registers to the infomobility service, he provides personal data including login information that are stored on the central system. It is preferable to avoid exchanging these data during the communications between the user and the highly distributed edge nodes in order to preserve the privacy of sensitive information, such as the identity and the current position of 


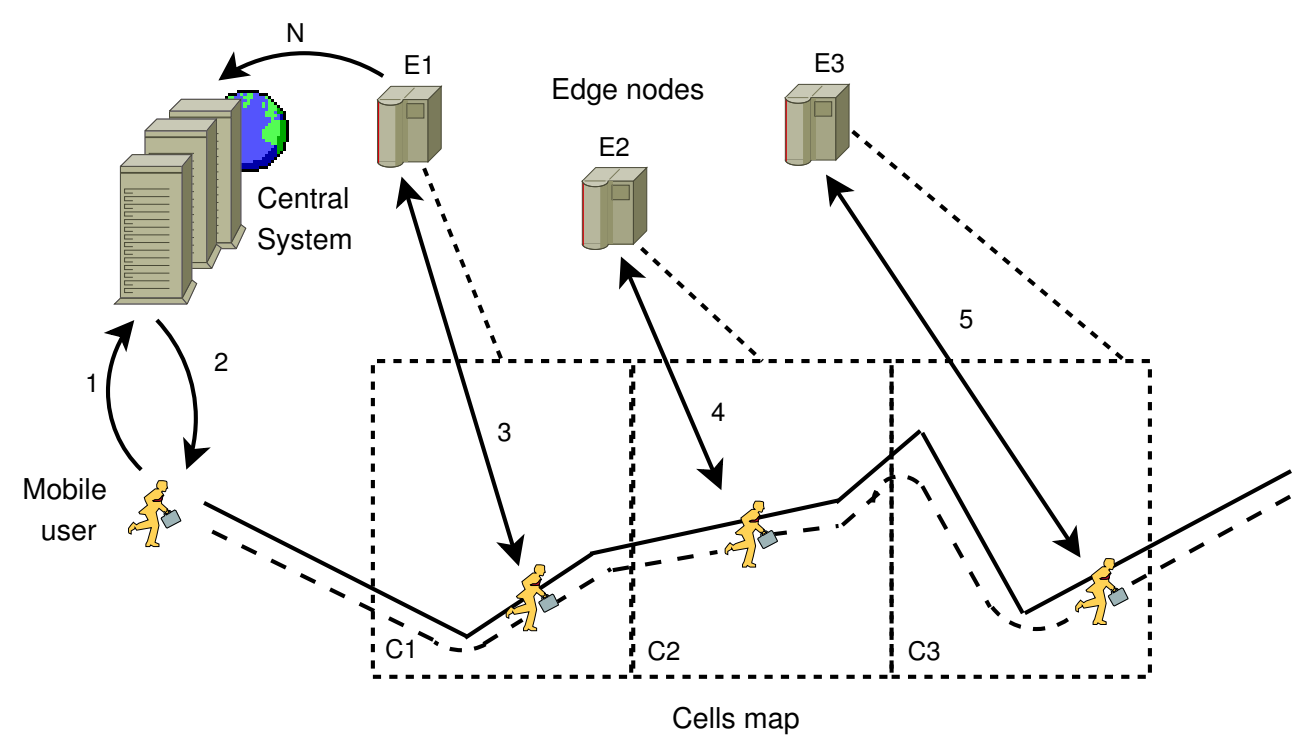

Figure 2. Dynamic description of infomobility architecture

the user. To this purpose, the central system associates each user with a token, that will be used for the communication with the edge nodes. Basically, a token contains the user ID, that may be just a numeric identifier associated to each user in a progressive way. A possible alternative could be the technique used by the FOAF project [7] that associates to each user a hash value calculated on his email address. Anyway, the important requirement to preserve the user privacy is to avoid the possibility to infer the user identity from the associated ID without accessing the information stored on the central system. Each token is signed with the central system private key so that the edge nodes are able to validate the user credentials. To reduce the risk of replay attacks, we limit the validity of the tokens by associating them with a limited time to live, so that a token is valid only for one itinerary. Moreover, we assume that the central system provides the user with a set of tokens, one for each edge node to contact, to restrict the validity period of a token to a single cell. A possible alternative is to communicate on a cryptographic channel, but this would introduce a significant overhead [1]. Hence, we prefer to discard this possibility also because the communications between the users and the edge nodes do not involve sensitive information.

\subsection{The distributed edge nodes}

In our architecture, each edge node manages and stores geo-referenced data concerning only one cell. The choice of partitioning the global territory into cells and assigning each of them to a different edge node is mo- tivated by scalability reasons. In this way, all the communications to and from users traveling across a specific cell are managed by a dedicated edge node, thus reducing the computational and network requirements and simplifying data management.

The notifications of the traveling users concern both critical communications of global relevance (e.g., detours, traffic jams, delays) and local information, such as points of interest. The distributed edge nodes have to operate filtering and aggregation functions on the user notifications for two main reasons: first, to extract and select meaningful information by all the received data; second, to reduce the traffic transmitted to the central system in case of critical updates that may affect the choices of the GIS system about the route planning. Figure 3 shows two different scenarios where the edge nodes operate data filtering and aggregation. On the left side of the figure, it is shown how the edge nodes may aggregate information coming from different users to identify congestion conditions on specific roads. Even if they do not host sophisticated GIS systems, the edge nodes are able to perform simple operations on geo-referenced data, such as the computation of the distance between two points located on the same road. This functionality allows the edge nodes to easily compute the average speed of a traveling user by comparing the information achieved through the user GPS receiver, such as the user's current position, with previously stored data. By aggregating information on the average speed of several users driving on the same road the edge node can compute the average speed of users. By comparing the average 


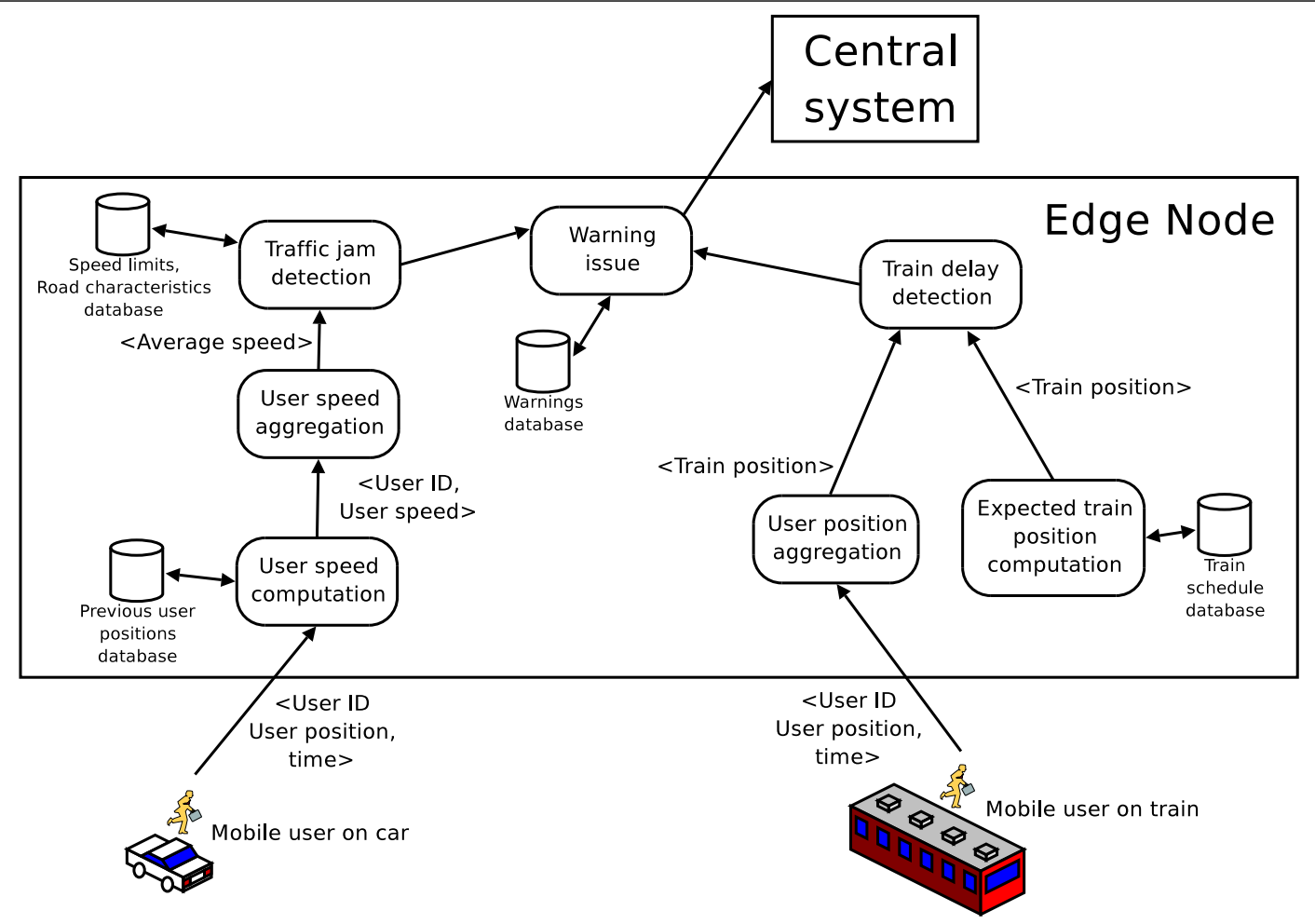

Figure 3. Edge nodes aggregation and filtering functions

speed with the expected speed due to road characteristics and speed limits, an edge node is able to identify possible traffic jams and generate a warning. If not already notified to the central system, the edge node transmits the warning about slowdowns in the traffic. In a similar way, comparing the position of a user traveling on a train with the information extracted from the train schedule allows to identify transportation delays, as shown on the right side of Figure 3. Indeed, the edge nodes may identify the train actual position by aggregating data on users location. Then, through a comparison with the expected position based on the train schedule, they are able to detect eventual delays that are notified through a warning to the central system.

Besides scalability issues, the partitioning of the geographical data among the edge nodes also helps improving system availability, by restricting to only one or few cells the effect of local failures. Moreover, simple redundancy techniques may be applied to improve the fault tolerance capabilities of the distributed architecture. We assume to replicate the information managed by each edge node on the nodes responsible for the near cells, so that they may take over in case of failure. This also simplifies hand-off operations between subsequent cells. The decision to replicate information on the close edge nodes is also motivated by the proximity of these nodes to the user in terms of Internet met- rics, that should allow the system to limit the variance in the response time in case of take over. The overhead to pay for applying redundancy techniques is related to the required periodic propagation of data from an edge node to the surrounding nodes. Propagation mechanisms based on lazy updates [10] are used to reduce this overhead, even if they introduce the risk of losing the latest update. However, this drawback is considered acceptable due to the non critical nature of the information stored on the edge nodes.

\subsection{The client device}

Even if the client device is not the focus of our architectural proposal, in this section we aim to describe the main features that are required on the client-side to exploit the infomobility service. In particular, it is important to note that the proposed distributed architecture for infomobility allows the user to rely on a thin client. Indeed, since the client device does not have to directly manage geo-referenced data for route planning, neither significant computational nor storage resources are required. Even more important from the user point of view, the infomobility system is able to always provide real-time updated information about the geographical area currently crossed by the user, thus avoiding the need for previous downloads and storage of large map 
sets.

Basically, the user only needs to dispose of a GPS receiver and a Web enabled device with specific features to access the infomobility Web services. It is possible to develop an ad-hoc application that inherits connectivity characteristics or to enrich a traditional Web browser with additional capabilities to avoid writing the application from scratch. A graphic and sound interface specifically designed for safe use while driving must be utilized for bidirectional information exchange between the system and the users. To this purpose, the communications reaching the user from the infomobility system have to be managed in a flexible way on the client-side, for example by using audio formats played by Java applets or AJAX applications. On the other hand, the system provides the user with a simple interface to send notifications to the edge nodes. To communicate information of global relevance, such as road accidents, traffic jams, road construction or detours, the interface will provide the users with single-click buttons specifying the type of information that has to be sent to the edge node along with the user current position. For the notification of less important POIs, such as petrol stations, hotels, restaurants, the interface will provide the user with the possibility to mark the point, store its coordinates, and complete the notification with additional information at a later time.

\section{Conclusions}

In this paper we address the issue of designing an architecture to support novel infomobility services. Our proposal provides support for services based on realtime interaction and cooperation among the mobile users that can travel by several different transportation means ranging from cars to trains to foot. The architecture design assumes thin clients that are not required to store a complete set of geographical data. We propose a novel distributed architecture based on two levels. A central system, that handles the most complex operations of route planning based on dynamic data, and a second level of distributed edge nodes that manage local geo-referenced data and interact with the mobile clients.

The analysis of the proposed architecture demonstrates that the two-level organization of nodes addresses the three main issues of infomobility: scalability, availability and user information privacy. Scalability is achieved through the highly distributed edge nodes. Availability is provided by adding redundancy in the information managed by the edge level. Finally, privacy is preserved by separating user identity from user location, with the user identity stored only on secure nodes in the central system.

\section{References}

[1] A. K. Agarwal and W. Wang. An experimental study on security protocols in wlans. Wireless Network Security, 2006. (Y. Xiao, X. Shen, and D.-Z. Du), Springer.

[2] V. Cardellini, E. Casalicchio, M. Colajanni, and P. S. Yu. The state of the art in locally distributed web-server systems. ACM Comput. Surv., 34(2):263-311, 2002.

[3] C.Bettini, X. S. Wang, and S. Jajodia. Protecting privacy against location-based personal identification. In Proc. of the 2nd VLDB Workshop on Secure Data Management, pages 185-199, Sep. 2005.

[4] R. K. Chellappa and R. G. Sin. Personalization versus privacy: An empirical examination of the online consumer's dilemma. Information Technology and Management, 6(2-3), April 2005.

[5] A. Cole, S. Duri, J. Munson, J. Murdock, and D. Wood. Adaptive service binding middleware to support mobility. In Proc. of the 23rd International Conference on Distributed Computing Systems Workshop (ICDCSW'03), pages 369-374, May 2003.

[6] Engadget. Uk drivers trust gps more than their own eyes, 2006. http://www.engadget.com/2006/04/20/ukdrivers-trust-gps-more-than-their-own-eyes/.

[7] Friend of a Friend project, 2006. http://www.foafproject.org/.

[8] Google Inc., 2006. http://earth.google.com.

[9] Google Inc., 2006. http://maps.google.com.

[10] J. Gray, P. Helland, P. O'Neil, and D. Shasha. The dangers of replication and a solution. In Proc. of the 1996 ACM SIGMOD International Conference on Management of Data, pages 173-182, Montreal, Canada, 1996.

[11] R. Hull, B. Kumar, D. Lieuwen, P. F. Patel-Schneider, A. Sahuguet, S. Varadarajan, and A. Vyas. Enabling context-aware and privacy-conscious user data sharing. In Proc. of 2004 IEEE International Conference on Mobile Data Management (MDM'04), 2004.

[12] Map-o-Rama, 2006. http://www.maporama.com/.

[13] L. Mazzucchelli and M. Pace. Extensible interfaces for mobile devices in an advanced platform for infomobility services. In Proc. of the working conference on Advanced visual interfaces, Gallipoli, IT, May 2004.

[14] C. Merlin and W. Heinzelman. A study of safety applications in vehicular networks. In Proc. of IEEE Int'l Conference onMobile Adhoc and Sensor Systems Conference, pages 102-109, Nov. 2005.

[15] P. Moraitis, E. Petraki, and N. I. Spanoudakis. Providing advanced, personalised infomobility services using agent technology. In Proc. of AI-2003, the 23rd SGAI International Conference on Innovative Techniques and Applications of Artificial Intelligence, Cambridge, UK, Dec. 2003.

[16] Tom Tom International, 2006. http://www.tomtom.com/plus/. 